\title{
Comportamento suicida em minorias sexuais: prevalência e fatores associados
}

\author{
Suicidal behavior in sexual minorities: prevalence and associated factors
}

Comportamiento suicida en minorías sexuales: prevalencia y factores associados

\begin{abstract}
Kauan Gustavo de Carvalho ${ }^{1 *}$, Lorena Uchoa Portela Veloso ${ }^{1}$, Mônica Madeira Marins Ferraz ${ }^{1}$, Claudete Ferreira de Souza Monteiro ${ }^{2}$, Nanielle Silva Barbosa ${ }^{1}$, Ana Caroliny de Barros Soares Lima ${ }^{1}$.
\end{abstract}

\section{RESUMO}

Objetivo: Analisar evidências científicas relacionadas à prevalência e fatores associados para o comportamento suicida em minorias sexuais. Métodos: Estudo de revisão integrativa da literatura, realizado nas bases de dados, Web of Science, MEDLINE/Pubmed, CINAHL e LILACS. Os descritores utilizados foram Pessoas LGBT, Suicídio, Ideação Suicida, Tentativa de Suicídio, Epidemiologia. Resultados: Obtevese uma amostra de quatorze artigos que atenderam aos critérios de inclusão estabelecidos com nível de evidência $2 \mathrm{~B}$. A população transexual obteve a maiores taxas de prevalência de ideação e tentativas suicidas com $67 \%$ e $35,5 \%$ respectivamente. $O$ fator de risco mais prevalente foi a discriminação e entre os fatores protetores destacou-se o apoio social e familiar. Considerações finais: As minorias sexuais apresentam-se mais propensas ao comportamento suicida, sendo necessárias a implementação de políticas preventivas com atenção específica para esse grupo.

Descritores: Pessoas LGBT, Suicídio, Prevalência e Fatores de Riscos.

\begin{abstract}
Objective: To analyze scientific evidence related to the prevalence and associated factors for suicidal behavior in sexual minorities. Methods: Integrative review of the literature, conducted in databases, Web of Science, MEDLINE / Pubmed, CINAHL and LILACS. The descriptors used were LGBT People, Suicide, Ideation, Suicide Attempt, Epidemiology. Results: We obtained a sample of fourteen articles that met the inclusion criteria established at level of evidence $2 \mathrm{~B}$. The transsexual population obtained the highest rates of suicidal ideation and suicide attempts with $67 \%$ and $35.5 \%$, respectively. The most prevalent risk factor was discrimination and among the protective factors, social and family support. Final considerations: Sexual minorities are more prone to suicidal behavior and are required to implement preventive policies with specific attention to this group.
\end{abstract}

Keywords: Sexual Minorities, Suicide, Prevalence and Risk factors.

\section{RESUMEN}

Objetivo: Analizar evidencias científicas relacionadas con la prevalencia y factores asociados con el comportamiento suicida en las minorías sexuales. Métodos: Estudio de revisión integrativa de la literatura, realizado en las bases de datos, Web of Science, MEDLINE / Pubmed, CINAHL y LILACS. Los descriptores utilizados fueron Personas LGBT, Suicidio, Ideación, Tentativa Suicida, Epidemiología. Resultados: Se

1Universidade Estadual do Piauí, Teresina, Piauí, Brasil. *E-mail: kauancarvalho2008@gmail.com

¿Universidade Federal do Rio de Janeiro. Rio de Janeiro, Rio de Janeiro, Brasil.

SUBMETIDO EM: 5/2019

ACEITO EM: 6/2019

PUBLICADO EM: 8/2019

REAS/EJCH | Vol.11(14) | e867 | DOI: https://doi.org/10.25248/reas.e867.2019 Página 1 de 9 
obtuvo una muestra de catorce artículos que atendieron a los criterios de inclusión establecidos con nivel de evidencia 2B. La población transexual obtuvo las mayores tasas de prevalencia de ideación e intentos suicidas con el $67 \%$ y el $35,5 \%$ respectivamente. El factor de riesgo más prevalente fue la discriminación y entre los factores protectores se destacó el apoyo social y familiar. Consideraciones finales: Las minorías sexuales se presentan más propensas al comportamento suicida, siendo necesarias para la implementación de políticas preventivas con atención específica para ese grupo.

Descriptores: Personas LGBT, El suicídio, Prevalencia y factores de riesgos.

\section{INTRODUÇÃO}

O suicídio é um ato intencional humano complexo e multifatorial que representa problema de saúde pública em todo o mundo. O comportamento suicida apresenta diversas manifestações associadas a pensamentos e atos auto lesivos que são classificados em: Ideação, plano, tentativa suicida e o suicídio propriamente dito (VELOSO C, et al., 2017).

Dados da Organização Mundial de Saúde (OMS) aponta que as taxas de suicídio aumentaram cerca de $60 \%$ nos últimos 45 anos, sendo considerada a $13^{\text {a }}$ causa mundial de morte da população (WHO, 2014). $O$ suicídio mata mais que os homicídios e as guerras juntos, com uma estimativa anual de um milhão de óbitos, correspondendo cerca de $1,4 \%$ do total de mortes no mundo (WHO, 2012).

O termo minorias sexuais englobam indivíduos que tem atrações sexuais/românticas pelo mesmo sexo ou de ambos os sexos, ou seja, aqueles que se identificam como Lésbicas, Gays, Bissexuais, Transgêneros, Transexuais, Travestis e Queer (LGBTQ). Estima-se que a taxa de tentativas suicidas entre os indivíduos LGBTQ é de 2 a 7 vezes maior do que o observado entre os heterossexuais (FRASER G, et al., 2018).

Entre os fatores associados ao comportamento suicida especificamente nesse grupo destacam-se os de ordem social e relacionais. É reconhecido que a população LGBTQ é exposta a ambientes hostis, onde vivenciam a discriminação e assédio devido a sua sexualidade, com particular relevância para o ambiente escolar, além de estarem mais sujeitos a níveis mais altos de violência, incluindo abuso sexual e físico, quando comparadas a pessoas heterossexuais (ALMAZAN EP, et al., 2014; BERGERON FA, et al., 2015).

Buscar reconhecer epidemiologicamente o comportamento suicida entre as minorias sexuais permite identificar com maior especificidade a heterogeneidade da prevalência e fatores associados, o que pode subsidiar a construção de políticas públicas mais eficazes, que incluam estratégias de prevenção e rastreamento de risco capazes de reduzir a morbidade e a mortalidade relacionadas ao suicídio nessa população vulnerável. Assim, este estudo tem como objetivo analisar evidências científicas relacionadas à prevalência e fatores associados para o comportamento suicida em minorias sexuais.

\section{METODOLOGIA}

Trata-se de revisão integrativa da literatura fundamentada em referencial teórico, conduzido por seis etapas de investigação: identificação do tema e seleção da hipótese ou questão de pesquisa; estabelecimento de critérios de inclusão e exclusão de estudos/amostragem ou busca na literatura; definição das informações a serem extraídas dos artigos selecionados/categorização dos estudos; avaliação dos estudos incluídos na revisão integrativa; interpretação dos resultados e apresentação do revisão/síntese do conhecimento (MENDES KDS, et al., 2008).

Para elaboração da questão de pesquisa foi utilizado a estratégia PICo (População: Pessoas LGBTQ; Interesse: Suicídio; Contexto: prevalência e fatores de riscos).

O resultando na seguinte questão: Quais as evidências científicas relacionadas a prevalência e fatores de riscos do comportamento suicida em minorias sexuais? (LOCKWOOD C, et al., 2017). 
Considerou-se critérios de inclusão: estudos primários disponíveis na íntegra nas bases de dados selecionadas. Excluiu-se estudos de abordagem qualitativas, revisão de literatura, duplicados, teses e dissertações.

A busca foi realizada por dois revisores, de forma simultaneamente e independente, no período de maio a julho de 2018, nas bases de dados: Literatura Latino-Americana e do Caribe em Ciências da Saúde (LILACS), Web of Science, Medical Literature Analysis and Retrieval Sistem Online (MEDLINE/Pubmed) e Cummulative Index to Nursing and Allied Health Literature (CINAHL). Utilizou-se descritores indexados nos Descritores em Ciências da Saúde (DeCS) e no Medical Subject Headings (MeSH), combinados por operadores boleanos "AND" e "OR" gerando expressões de busca conforme Quadro 1.

Quadro 1 - Expressões de busca aplicadas às bases de dados conforme descritores selecionados mediante estratégia PICO. Teresina, PI, 2018.

\begin{tabular}{|c|c|c|c|}
\hline \multicolumn{4}{|c|}{ Descritores em Ciências da Saúde - DECS } \\
\hline \multicolumn{2}{|r|}{ DESCRITORES } & BASE DE DADOS & EXPRESSÃO FINAL DE BUSCA \\
\hline $\mathbf{P}$ & Pessoas LGBT & \multirow[t]{3}{*}{ LILACS } & \multirow{3}{*}{$\begin{array}{l}\text { Pessoas LGBT AND Suicídio/Idealização } \\
\text { OR Tentativa Suicida AND Epidemiologia }\end{array}$} \\
\hline I & $\begin{array}{l}\text { Suicídio/ldeação e } \\
\text { Tentativa suicida }\end{array}$ & & \\
\hline Co & Epidemiologia & & \\
\hline \multicolumn{4}{|c|}{ Medical Subject Headings - MESH e list CINAHL } \\
\hline \multicolumn{2}{|r|}{ DESCRITORES } & BASE DE DADOS & EXPRESSÃO FINAL DE BUSCA \\
\hline $\mathbf{P}$ & Sexual Minorities & MEDLINE/Pubmed & $\begin{array}{l}\text { Sexual Minorities OR Gender minorities } \\
\text { AND Suicide OR Suicide attempt AND } \\
\text { Prevalence OR Epidemiology }\end{array}$ \\
\hline I & Suicide/ Suicide attempt & Web of Science & $\begin{array}{l}\text { Sexual minorities OR Gender minorities } \\
\text { AND Suicide OR Suicide attempt OR } \\
\text { Suicide ideation OR Suicidality AND } \\
\text { Prevalence OR Epidemiology OR Risk } \\
\text { factors }\end{array}$ \\
\hline $\begin{array}{l}\text { C } \\
\text { O }\end{array}$ & Epidemiology & CINAHL & $\begin{array}{l}\text { Sexual minorities OR GLBT persons OR } \\
\text { Transgender persons AND Suicide OR } \\
\text { Suicide attempt OR Suicide ideation AND } \\
\text { Epidemiology OR Epidemiology Research }\end{array}$ \\
\hline
\end{tabular}

Fonte: Dados da pesquisa, 2018.

Para coleta de dados utilizou-se instrumento, elaborado pelos autores deste estudo, contendo informações acerca do ano de publicação, país de origem, detalhamento do método, prevalência e fatores associados.

Para a classificação do nível de evidência utilizou-se a recomendação do Oxford Centre for Evidencebased medicine (DURIEUX N, et al., 2013).

No primeiro momento foi realizada a busca nas bases de dados e exclusão dos duplicados. Em seguida foi realizada revisão do título e resumo para inclusão dos estudos primários pertinentes.

No momento seguinte, e de forma também independente, houve a leitura do texto completo dos artigos. As discordâncias foram resolvidas por meio de discussão com terceiro revisor (Figura 1). 
Figura 1 - Estabelecimento dos critérios de inclusão e exclusão dos artigos nas bases de dados LILACS, Web Of Science, CINAHL e MEDLINE/Pubmed Teresina, PI, 2018.

№ de relatos identificados nos bancos LILACS (11), Web Of Science

(260), CINAHL (13), MEDLINE/Pubmed (12) N= 296

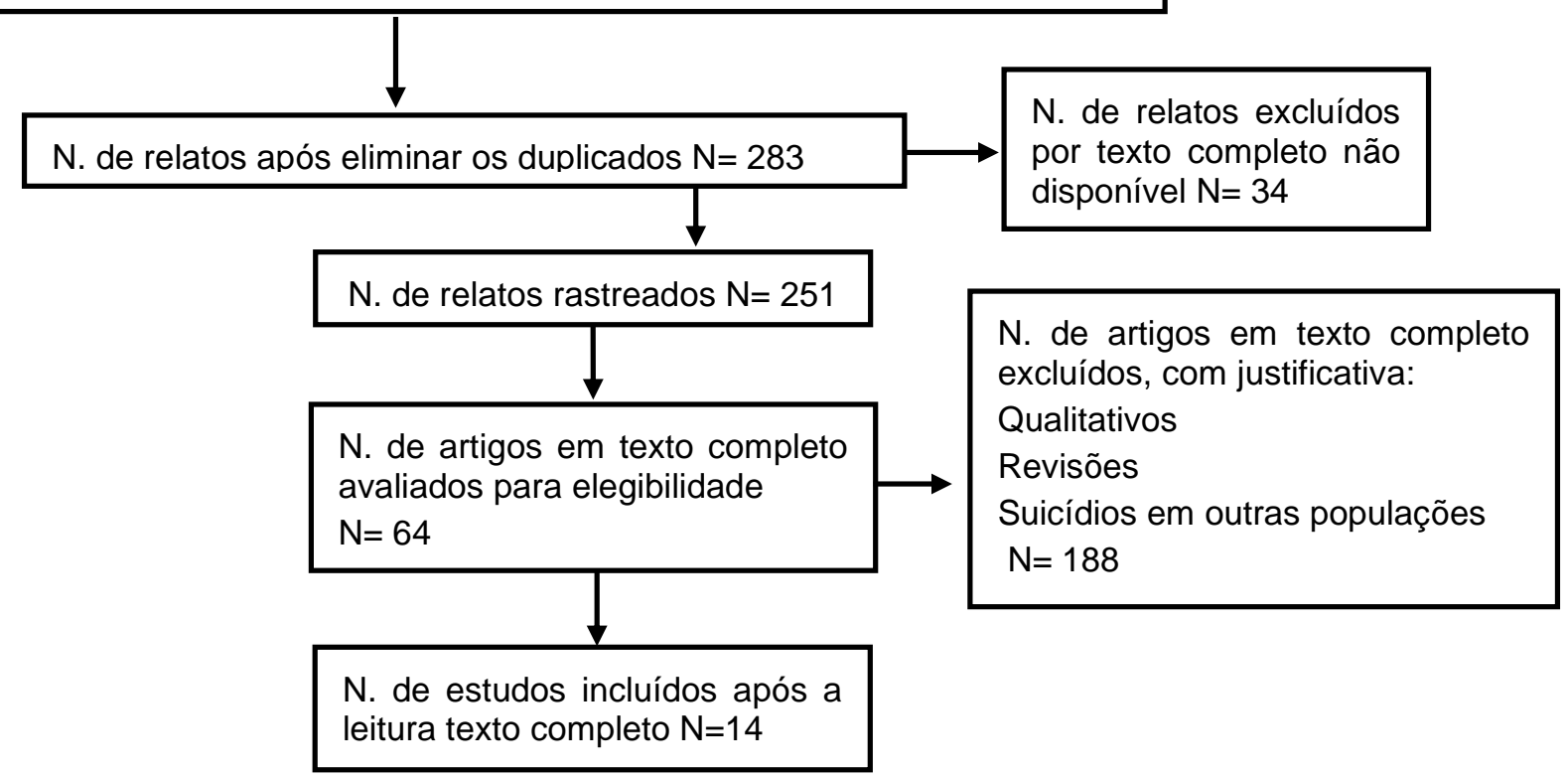

Fonte: Dados da pesquisa, 2018.

\section{RESULTADOS}

Foram encontrados nas bases de dados 296 artigos, sendo 11 na LILACS, 260 Web Of Science (260), 13 na Cummulative Index to Nursing and Allied Health Literature (CINAHL) e 12 Medical Literature Analysis and Retrieval Sistem Online (MEDLINE/Pubmed). Após a eliminação dos duplicados restaram 283 artigos.

Em seguida foram excluídos 34 artigos por texto completo não disponível, restando 251 artigos, que após a leitura de título e resumo, foram excluídos aqueles que eram de cunho qualitativos, de revisões e suicídios em outras populações, restando apenas 64 artigos para análise do texto completo para elegibilidade. No momento seguinte, e de forma também independente, houve a leitura do texto completo dos artigos restando apenas 14 artigos (Figura 1).

Os 14 artigos selecionados foram pulicados no período entre 2012 a 2018, com destaque para o ano de 2016 com 5 (35,7\%) publicações. Quanto ao local de pesquisa, houve predomínio da América do Norte com $9(64,2 \%)$. Ressalta-se que não foi encontrado nenhum artigo brasileiro em todas as etapas da pesquisa (Quadro 2).

Em relação ao delineamento do método, abordagem metodológica mais frequente foi o estudo de coorte com $8(57,1 \%)$ e as amostras dos estudos variaram de 68 a 146621 participantes. Quanto ao nível de evidencia observou-se que o nível 2 B prevaleceu com 8 (57,1\%) publicações (Quadro 2).

O Quadro 2 traz a síntese das prevalências e fatores associados. A população mais prevalente em relação a ideação suicida e tentativa foi a transexual com uma taxa de $67 \%$ e $35,5 \%$ respectivamente e a menor foi em homossexuais com $22 \%$ para ideação e $17 \%$ para tentativas.

Em relação aos fatores de riscos o que mais se destacou foi a discriminação citada em 10 artigos $(71,4 \%)$. Entre os fatores protetores que tiveram mais predomínio foram apoio social e familiar com 10 $(71,4 \%)$ dos artigos.

Embora não apontado em muitos artigos, cabe destacar o reconhecimento da identidade de gênero nos documentos oficiais como fator protetor para o comportamento suicida. 
Quadro 2 - Distribuição das referências por autor, ano de publicação, país de origem, área de atuação dos autores, nível de evidencia e delineamento do método. Teresina, PI, 2018.

\begin{tabular}{|c|c|c|c|c|}
\hline Autor/Ano & País & NE & Delineamento do método & Prevalência/fatores associados \\
\hline Bauer GR et al. (2015) & Canada & 1B & $\begin{array}{l}\text { Tipo de estudo: Randomizado controlado } \\
\text { Amostra: } 380 \text { pessoas transexuais com idade } \\
\text { acima de } 16 \text { anos em Ontário, Canadá }\end{array}$ & $\begin{array}{l}\text { Prevalência: Ideação e tentativa suicida nos últimos } 12 \\
\text { meses respetivamente: } 35,1 \% \text { e } 11,2 \% \text {. } \\
\text { Fatores de risco: Transfobia e depressão. } \\
\text { Fatores de proteção: Apoio social, Reconhecimento da } \\
\text { identidade de gênero. }\end{array}$ \\
\hline Blosnich JR et al. (2012) & EUA & $2 \mathrm{~B}$ & $\begin{array}{l}\text { Tipo de estudo: Estudo de coorte Amostra: } \\
61 \text { minorias sexuais e } 1639 \text { heterossexuais de } \\
\text { instituições educacionais }\end{array}$ & $\begin{array}{l}\text { Prevalência: Ideação suicida em minorias sexuais nos } \\
\text { últimos } 12 \text { meses: } 11,48 \% \text {. Fatores de risco: problemas } \\
\text { psiquiátricos. Fatores de proteção: apoio social e } \\
\text { familiar. }\end{array}$ \\
\hline Bouris A et al. (2016) & EUA & $2 \mathrm{~B}$ & $\begin{array}{l}\text { Tipo de estudo: Estudo de coorte } \\
\text { Amostra: } 1.907 \text { estudantes em escolas } \\
\text { secundárias públicas de Chicago no ano } 2011\end{array}$ & $\begin{array}{l}\text { Prevalência: Ideação suicida e tentativa em minorias } \\
\text { sexuais nos últimos } 12 \text { meses respectivamente: } 27,95 \text { e } \\
\text { 29,92\%. Fatores de risco: Violência, abuso sexual, } \\
\text { discriminação, bullying e ciberbullying, depressão. } \\
\text { Fatores de proteção: Apoio escolar. }\end{array}$ \\
\hline $\begin{array}{l}\text { Duncan DT, Hatzenbuehler } \\
\text { ML (2014) }\end{array}$ & EUA & $2 \mathrm{~B}$ & $\begin{array}{l}\text { Tipo de estudo: Estudo de Coorte. } \\
\text { Amostra: } 1292 \text { alunos de } 9 \text { a } 12 \text { anos de } \\
\text { escolas públicas de Boston. }\end{array}$ & $\begin{array}{l}\text { Prevalência: Ideação suicida em minorias sexuais nos } \\
\text { últimos } 12 \text { meses: } 32,35 \% \text {. Tentativa de suicídio em } \\
\text { minorias sexuais nos últimos } 12 \text { meses: } 16,67 \% \text {. } \\
\text { Fatores de risco: Violência e crimes de ódio. } \\
\text { Fatores de proteção: Apoio social e familiar. }\end{array}$ \\
\hline $\begin{array}{l}\text { Hatzenbuehler ML, Keyes } \\
\text { KM (2013) }\end{array}$ & EUA & $1 \mathrm{~B}$ & $\begin{array}{l}\text { Tipo de estudo: Estudo randomizado } \\
\text { controlado. } \\
\text { Amostra: Um total de } 31.852 \text { estudantes do } \\
11^{\circ} \text { ano da escola pública. }\end{array}$ & $\begin{array}{l}\text { Prevalência: Tentativa de suicídio em gays e lésbicas } \\
\text { nos últimos } 12 \text { meses: } 31,08 \% \text { Tentativa de suicídio em } \\
\text { bissexuais nos últimos } 12 \text { meses: } 25,65 \text {. Fatores de } \\
\text { risco: Violência e bullying. Fatores de proteção: Apoio } \\
\text { escolar. }\end{array}$ \\
\hline Kim GH et al. (2016) & $\begin{array}{l}\text { Coréia do } \\
\text { Sul }\end{array}$ & 2B & $\begin{array}{l}\text { Tipo de estudo: Estudo de Coorte } \\
\text { Amostra: } 146.621 \text { estudantes com idades } \\
\text { entre } 12 \text { e } 17 \text { anos }\end{array}$ & $\begin{array}{l}\text { Prevalência: ideação de suicídio nos últimos } 12 \text { meses: } \\
\text { lésbicas }(45,9 \%) \text {, gays ( } 22 \%) \text {. Tentativa de suicídio nos } \\
\text { últimos } 12 \text { meses: lésbicas ( } 28,4 \%) \text {, gays ( } 17 \%) \text {. } \\
\text { Fatores de risco: Violência e discriminação. } \\
\text { Fatores de proteção: Apoio familiar e escolar. }\end{array}$ \\
\hline Kohlbrenner V et al. (2016) & Suécia & $2 \mathrm{~B}$ & $\begin{array}{l}\text { Tipo de estudo: Estudo de Coorte } \\
\text { Amostra: } 400 \text { participantes em } 2012 .\end{array}$ & $\begin{array}{l}\text { Prevalência: ideação de suicídio e tentativa em } \\
\text { minorias sexuais nos últimos } 12 \text { meses respectivamente: } \\
26,8 \% \text { e } 9 \% \text {. Fatores de risco: Violência, discriminação. } \\
\text { Fatores de proteção: Apoio social e familiar. }\end{array}$ \\
\hline
\end{tabular}

REAS/EJCH | Vol.11(14) | e867 | DOI: https://doi.org/10.25248/reas.e867.2019 Página 5 de 9 


\begin{tabular}{|c|c|c|c|c|}
\hline Kralovec K et al. (2014) & Áustria & $1 \mathrm{~B}$ & $\begin{array}{l}\text { Tipo de estudo: Estudo randomizado } \\
\text { controlado } \\
\text { Amostra: } 358 \text { austríacos lésbicos, gays e } \\
\text { bissexuais. }\end{array}$ & $\begin{array}{l}\text { Prevalência: ideação e tentativa de suicídio em } \\
\text { minorias sexuais nos últimos } 12 \text { meses respectivamente: } \\
\text { 17\% e 14\%. Fatores de risco: Discriminação e religião. } \\
\text { Fatores de proteção: Apoio social e familiar. }\end{array}$ \\
\hline Levasseur MT et al. (2013) & EUA & $2 \mathrm{~B}$ & $\begin{array}{l}\text { Tipo de estudo: Estudo de Coorte } \\
\text { Amostra: } 11.887 \text { alunos do } 9^{\circ} \text { ao } 12^{\circ} \text { ano, em } \\
2009 \text {, para examinar a associação de } \\
\text { identidade sexual e bullying com tentativa de } \\
\text { suicídio. }\end{array}$ & $\begin{array}{l}\text { Prevalência: Tentativa de suicídio em minorias sexuais } \\
\text { nos últimos } 12 \text { meses: } 9,9 \% \text {. } \\
\text { Fatores de risco: Violência e bullying. Fatores de } \\
\text { proteção: Apoio escolar. }\end{array}$ \\
\hline Lytle MC et al. (2018) & EUA & 2B & $\begin{array}{l}\text { Tipo de estudo: Estudo de Coorte } \\
\text { Amostra: } 21.247 \text { jovens adultos entre } 18 \text { e } 30 \\
\text { anos relataram identidade sexual e relações de } \\
\text { importância religiosa. }\end{array}$ & $\begin{array}{l}\text { Prevalência: ideação de suicídio em minorias sexuais } \\
\text { negros nos últimos } 12 \text { meses: } 10,1 \% \text {. } \\
\text { Fatores de risco: Discriminação e religião. Fatores de } \\
\text { proteção: Apoio social e familiar. }\end{array}$ \\
\hline Mustansk B e Liu RT (2013) & EUA & 1B & $\begin{array}{l}\text { Tipo de estudo: Estudo randomizado } \\
\text { controlado } \\
\text { Amostra: } 248 \text { jovens LGBT. }\end{array}$ & $\begin{array}{l}\text { Prevalência: Tentativa de suicídio em minorias sexuais } \\
\text { nos últimos } 12 \text { meses: } 31,6 \% \text {. Fatores de risco: } \\
\text { Discriminação, Depressão. } \\
\text { Fatores de proteção: Apoio social e familiar }\end{array}$ \\
\hline Smith BC et al. (2016) & EUA & 1B & $\begin{array}{l}\text { Tipo de estudo: Estudo randomizado } \\
\text { controlado } \\
\text { Amostra: } 68 \text { jovens LGB traumatizados }\end{array}$ & $\begin{array}{l}\text { Prevalência: Ideação e tentativa de suicídio em } \\
\text { minorias sexuais nos últimos } 12 \text { meses respectivamente: } \\
54,4 \% \text { e } 32,4 \% \text {. } \\
\text { Fatores de risco: Discriminação, uso de substancias } \\
\text { psicoativas e depressão } \\
\text { Fatores de proteção: Apoio social e familiar. }\end{array}$ \\
\hline Suen YT et al. (2018) & China & 1B & $\begin{array}{l}\text { Tipo de estudo: Estudo randomizado } \\
\text { controlado } \\
\text { Amostra: } 106 \text { pessoas transexuais no ano de } \\
2012 \text { a } 2013 \text {. }\end{array}$ & $\begin{array}{l}\text { Prevalência: Ideação e tentativa de suicídio em } \\
\text { minorias sexuais nos últimos } 12 \text { meses } \\
\text { respectivamente: } 67,0 \% \text { e } 35,5 \% \text {. Fatores de risco: } \\
\text { Discriminação, renda mensal e Depressão. Fatores de } \\
\text { proteção: Apoio social e familiar. }\end{array}$ \\
\hline Woodhead C et al. (2016) & Reino Unido & $2 \mathrm{~B}$ & $\begin{array}{l}\text { Tipo de estudo: Estudo de Coorte. } \\
\text { Amostra: } 8455 \text { pessoas de cidades diferentes } \\
\text { do Reino Unido }\end{array}$ & $\begin{array}{l}\text { Prevalência: Ideação de suicídio nos últimos: } 51,5 \% \text {. } \\
\text { Fatores de risco: Discriminação, trauma na infância e } \\
\text { depressão. Fatores de proteção: Apoio social e familiar }\end{array}$ \\
\hline
\end{tabular}

Fonte: Dados da pesquisa, 2018.

Legenda: NE: Nivel de evidência.

REAS/EJCH | Vol.11(14) | e867 | DOI: https://doi.org/10.25248/reas.e867.2019 Página 6 de 9 


\section{DISCUSSÃO}

Observa-se um interesse recente pelas publicações relacionadas ao comportamento suicida em minorias sexuais, com intensificação no ano de 2016. Tal fato pode estar relacionado a publicação, em 2015, de declaração conjunta sem precedentes pedindo o fim da violência e discriminação contra minorias sexuais, feita pela Organização das Nações Unidas (ONU) juntamente com mais 12 entidades (ONU, 2015).

Em 2016, nos Estados Unidos, pais com maioria nas publicações, divulgou o guia c "Vivendo livres e igualmente", que contém uma série de recomendações aos países voltadas a proteger a população LGBTQ e intersexual (HUSSEIN ZRA, 2016).

\section{Prevalência do comportamento suicida}

As minorias sexuais apresentam maiores prevalências de comportamento suicida quando comparadas com populações heterossexuais. A prevalência de ideação suicida na população LGBTQ variou de $22 \%$ a $67 \%$, enquanto que na população geral citadas nos estudos variou de 3,48\% a 23,8\% (KIM GH, et al., 2016, SUEN YT, et al., 2018; BLOSNICH JR, et al., 2012). Tais dados corroboram com estudo realizado nos Estados Unidos, onde as minorias sexuais obtiveram 3.76 mais chances de ideação suicida quando comparada com população geral (BLOSNICH JR, et al., 2016). Entre os fatores associados a ideação suicida nesse grupo destaca-se problemas de saúde mental e menor suporte social e emocional (KOHLBRENNER V, et al., 2016; SMITH BC, et al. 2016).

As tentativas de suicídio variaram percentuais de $17 \%$ a $35,5 \%$ em minorias sexuais, enquanto em indivíduos heterossexuais obtiveram prevalências de $1 \%$ a $17 \%$ com aquele grupo associado significativamente a maiores chances de tentativas de suicídio ( $\mathrm{KIM} \mathrm{GH}$, et al., 2016; BOURIS A, et al., 2016; SUEN YT, et al., 2018; KRALOVEC K, et al., 2014). Estudo randomizado controlado com essa população descobriu que os participantes com histórico de tentativa de suicídio foram 10 vezes mais propensos a fazer outra tentativa de suicídio no ano seguinte do que aqueles que não tinham tentado anteriormente (MUSTANSKI B e LIU RT, 2015).

A população transexual obteve as maiores taxas de prevalência de tentativas de suicídio variando de 11,2 a $35,5 \%$ ao longo da vida, nos países como EUA e China, respectivamente. Outros estudos em outros países com pessoas transexuais também relataram altas taxas de tentativa de suicídio: $16 \%$ nas Filipinas, 22\% em Tailândia e 32\% no Japão (BAUER GR, et al., 2015; SUEN YT, et al., 2018; TERADA S, et al., 2011). Vários estudos apontam que jovens transgêneros são mais vulneráveis ao abuso de gênero, fator que estaria associado esse comportamento (BAUER GR, et al., 2015; TSYPES A, et al., 2016; SUEN YT, et al., 2018).

Em relação ao sexo masculino, os homens gays foram mais propensos a relatar tentativa de suicídio nos últimos 12 meses do que homens heterossexuais, onde as mulheres de forma geral relatam mais tentativas do que os homens. Embora o sexo feminino seja duas vezes mais provável relatar tentativas suicidas do que o sexo masculino, estes têm mais êxitos nas tentativas, logo maiores números de suicídios consumados, cerca de 3 a 5 vezes maiores que as mulheres (BLOSNICH JR, et al., 2012).

\section{Fatores associados ao comportamento suicida}

As minorias sexuais quando comparada a população em geral possuem fatores riscos e de proteção semelhantes para comportamento suicida como: depressão, discriminação, uso de substâncias psicoativas, insegurança escolar e suporte inadequado, seja familiar ou social (BOURIS A, et al., 2016; SMITH BC, et al., 2016).

A religião muitas vezes é percebida como um fator de proteção especialmente na população geral. Porém, para indivíduos LGBTQ podem apresentar-se como fator de risco, visto que muitas religiões proíbem atividades sexuais com pessoas do mesmo sexo e a veem como pecaminosa ou não natural.

Tais crenças podem induzir a uma "homofobia internalizada", na qual o indivíduo incorpora crenças religiosas que veem a atração sexual pelo mesmo sexo com algo negativo, o que pode ser associado a desfechos negativos para a saúde mental, pelo conflito entre identidades religiosas e sexuais, que podem 
resultar em vergonha, depressão e ideação suicida. As denominações religiosas mais frequentes foram o catolicismo, seguido pelo protestantismo (LYTLE MC, et al., 2015; MUSTANSKI B e LIU RT, 2015).

As relações familiares em que ocorrem reações de rejeição foram apontadas como fator de risco, quando comparadas com aquelas em que houve apoio familiar (LYTLE MC, et al., 2015; MUSTANSKI B e LIU RT, 2015).

As relações familiares podem ser interrompidas, principalmente na época da revelação ou descoberta da identidade sexual, em razão da rejeição familiar, o que leva a muitos desses indivíduos ficarem desabrigados, outro fator de risco para o comportamento suicida (HATZENBUEHLER ML e KEYES KM, 2013).

Principalmente, quando os indivíduos se distanciam do que família esperava para o seu futuro, isso ocorre com maior prevalência em indivíduos transgêneros, contemplando as maiores taxas de rejeição familiar com cerca de 57\% (TERADA S, et al., 2011; BAUER GR, et al., 2015, SUEN YT, et al., 2018).

Em contraste, quando os pais aceitam é provável que o adolescente se sinta mais seguros e protegidos (DUNCAN DT e HATZENBUEHLER ML, 2014; KRALOVEC K, et al., 2014; BAUER GR, et al., 2015; KIM $\mathrm{GH}$, et al., 2016). De fato, jovens LGBTQ adultos que vieram de famílias altamente aceitáveis eram menos prováveis ter pensamentos suicidas. Com maiores taxas de autoestima, apoio social e saúde do que aqueles que não tiveram aceitação (SMITH BC, et al. 2016; WOODHEAD C, et al., 2016).

O apoio familiar é essencial na proteção contra as ameaças externas, visto que a maioria das minorias sexuais sofrerem os mais altos níveis de violência, incluindo abuso sexual e físico, quando comparados com a população heterossexual (KOHLBRENNER V, et al., 2016; SMITH BC, et al. 2016).

A história de abuso familiar ou experiência de abuso sexual pode elevar o risco de suicídio e depressão em minorias sexuais (LYTLE MC, et al., 2015; BOURIS A, et al., 2016; SUEN YT, et al., 2018). Também são mais propensas a ter piores resultados de saúde, bem como níveis mais elevados de sofrimento psicológico, incluindo ansiedade e depressão, que muitas vezes resultam na dificuldade de lidar com a sexualidade (WOODHEAD C, et al., 2016; ESPELAGE DL, et al., 2018).

A população LGBTQ tendem a ser mais expostos a ambientes hostis. Um dos contextos mais relevante seria o ambiente escolar, visto que é o principal local de práticas bullying, que incluiu desde ameaças verbais, abuso físico, até ameaças de morte. Logo, a incidência de discriminação tende a aumentar em consonância com aparecimento dos sintomas depressivos que por sua vez elevam o comportamento suicida (HATZENBUEHLER ML e KEYES KM, 2013; LEVASSEUR MT, et al., 2013; BOURIS A, et al., 2016).

Além disso, as escolas têm papel fundamental na segurança e proteção dessa população, bem como na elaboração de programas e políticas anti bullying que visam melhorar a acessibilidade nessa população. Em contrapartida em ambientes que não ofertam apoio escolar tiveram cerca de $31,08 \%$ tentativas de suicídio em comparação aos que tinham de forma efetiva com apenas $16,67 \%$, retratando com o apoio escolar é essencial na redução do comportamento suicida, bem como na redução das taxas de depressão, uso de drogas, e evasão escolar (KIM GH, et al., 2016; WOODHEAD C, et al., 2016).

\section{CONSIDERAÇÕES FINAIS}

Percebe-se que as minorias sexuais são mais propensas ao comportamento suicida comparadas a população geral, com destaque a população transexual que apresenta maiores prevalências. Em relação aos fatores de riscos a discriminação foi a que obteve o maior impacto para esse comportamento. Além, do apoio social e familiar considerados fatores protetores para diminuir essa prevalência. Tais dados apontam para uma necessária reflexão das políticas de prevenção do comportamento nesse grupo especifico, que possam incluir estratégias adequadas aos fatores de risco e protetores identificados. Melhorando os métodos de triagem e fortalecendo a promoção de qualidade de vida; além de informar e sensibilizar a sociedade de que o suicídio é um problema de saúde pública que pode ser prevenido, muitas vezes com a identificação dos fatores de riscos, com intuito de reduzir a morbimortalidade dessa população vulnerável. 


\section{REFERÊNCIAS}

1. ALMAZAN EP, et al. Measures of Sexual Minority Status and Suicide Risk among Young Adults in the United States. Rev. Arch Suicide Res. 2014;18(3):274-81. doi: 10.1080/13811118.2013.824832.

2. BAUER GR, et al. Intervenable factors associated with suicide risk in transgender persons: a respondent driven sampling study in Ontario, Canada. Rev. BMC Public Health. 2015;15(2):525. doi: 10.1186/s12889-015-1867-2.

3. BERGERON FA, et al. The role of parental support in the relationship between homophobic bullying, internalized homophobia and psychological distress among sexual-minority youths (SMY): a moderated mediation approach. Rev. Sante Ment Que. 2015;40(3):109-27.

4. BLOSNICH JR, et al. Suicidal Ideation Among Sexual Minority Veterans: Results From the 2005-2010 Massachusetts Behavioral Risk Factor Surveillance Survey. Rev. American Journal of Public Health. 2012;102 Supl. 1: S44-7. doi: 10.2105 / JPH.2011.300565.

5. BLOSNICH JR, et al. Mental Health of Transgender Veterans in US States With and Without Discrimination and Hate Crime Legal Protection. Rev. Am J Public Health. 2016;106(3):534-40. doi: 10.2105/AJPH.2015.302981.

6. BOURIS A, et al. Effects of Victimization and Violence on Suicidal Ideation and Behaviors Among Sexual Minority and Heterosexual Adolescents. Rev. LGBT Health. 2016;3(2):153-61. doi: 10.1089 / lgbt.2015.0037.

7. DUNCAN DT, HATZENBUEHLER ML. Lesbian, Gay, Bisexual, and Transgender Hate Crimes and Suicidality Among a Population-Based Sample of Sexual-Minority Adolescents in Boston. 2014;104(2):272-8. doi: 10.2105/AJPH.2013.301424

8. DURIEUX N, et al. Médecine Factuelle: la hiérarchisation des preuves par le Centre for Evidence-Based Medicine d'Oxford. Rev Med Liège. 2013;68(12):644-649.

9. ESPELAGE DL, et al. Peer Victimization and Dating Violence Among LGBTQ Youth: The Impact of School Violence and Crime on Mental Health Outcomes. Rev. Youth Violence and Juvenile Justice. 2018;6(2):156-173.

10. FRASER G, et al. Non-Suicidal Self-Injury, Sexuality Concerns, and Emotion Regulation among Sexually Diverse Adolescents: A Multiple Mediation Analysis. Rev. Arch Suicide Res. 2018;22(3):432-452. doi: 10.1080/13811118.2017.1358224.

11. HATZENBUEHLER ML, KEYES KM. Inclusive Anti-bullying policies and Reduced Risk of Suicide Attempts in Lesbian and Gay Youth. Rev. 2013;53 Supl (1):S21-6. doi: 10.1016/j.jadohealth.2012.08.010.

12. HUSSEIN ZRA. Living free and equal. What states are doing to tackle violence and discrimination against lesbian, gay, bisexual, transgender and intersex people. United Nations High Commissioner for Human Rights. 2016.

13. KIM GH, AHN HS, KIM HJ. Type of sexual intercourse experience and suicidal ideation, plans, and attempts among youths: a cross-sectional study in South Korea. Rev. Bmc Public Health. 2016;16(1):1229.

14. KOHLBRENNER V, et al. Perceived Discrimination Is an Independent Risk Factor for Suicidal Ideation among sexual and Gender Minorities in Nepal. Rev. PLoS One. 2016;11(7):0159359. doi: 10.1371/journal.pone.0159359.

15. KRALOVEC K, et al. Religion and suicide risk in lesbian, gay and bisexual Austrians. Rev. Journal of Religion \& Health. 2014;53(2):413-423. doi: 10.1007 / s10943-012-9645.

16. LEVASSEUR MT, et al. Intersecting Identities and the Association Between Bullying and Suicide Attempt Among New York City Youths: Results From the 2009 New York City Youth Risk Behavior Survey. Rev. American Journal, of Public Health, 2013;103(6):1082-9. doi: 10.2105/AJPH.2012.300994.

17. LOCKWOOD C, et al. Chapter 2: Systematic reviews of qualitative evidence. In: Aromataris E, Munn Z (Editors). Joanna Briggs Institute Reviewer's Manual. The Joanna Briggs Institute [Internet]. 2017.

18. LYTLE MC, et al. Associations of racial/ethnic identities and religious affiliation with suicidal ideation among lesbian, gay, bisexual, and questioning individuals. Rev. J Affect Disord. 2015; 178:39-45. doi: 10.1016 / j.jad.2014.07.039.

19. MENDES KDS, et al. Revisão integrativa: método de pesquisa para a incorporação de evidências na saúde e na enfermagem. Rev. Texto \& Contexto Enfermagem. Florianópolis. 2008;17(4):758-64.

20. MUSTANSKI B, LIU RT. A longitudinal study of predictors of suicide attempts among lesbian, gay, bisexual, and transgender youth. Rev. Arch Sex Behav. 2013;42(3):437-48. doi: 10.1007/s10508-012-0013-9.

21. ONU, United Nations. 2015. Statement on ending violence and discrimination against LGBT and intersex persons. Disponível em: www.ohchr.org/EN/Issues/Discrimination/Pages/JointLGBTIstatement.aspx. Acesso em: 02 jul. 2018.

22. SMITH BC, et al. PTSD, Depression, and Substance Use in Relation to Suicidality Risk among Traumatized Minority Lesbian, Gay, and Bisexual Youth. Rev. Arch Suicide Res. 2016;20(1):80-93.

23. SUEN YT, et al. Mental Health of Transgender People in Hong Kong: A Community-Driven, Large-Scale Quantitative Study Documenting Demographics and Correlates of Quality of Life and Suicidality. Rev. Journal of Homosexuality. 2018; 65(8):10931113. doi: 10.1080/00918369.2017.1368772.

24. TERADA S, et al. Suicidal ideation among patients with gender identity disorder. Rev. Psychiatry Res. 2011;190(1):159-62. doi: 10.1016/j.psychres.2011.04.024 Hong Kong Law Journal.

25. TSYPES A, et al. Non-suicidal self-injury and suicidal thoughts and behaviors in heterosexual and sexual minority young adults. Rev. Comprehensive Psychiatry. 2016; 65:32-43. doi: 10.1016/j.comppsych.2015.09.012.

26. VELOSO C, et al. Violência autoinfligida por intoxicação exógena em um serviço de urgência e emergência. Rev Gaúcha Enferm. 2017;38(2):66-187.

27. WHO, World Health Organization. 2012. Ação de saúde pública a prevenção do suicídio: um quadro. Geneva, 2012 Disponível em: http://apps.who.int/iris/bitstream/10665/75166/1/9789241503570 eng. Acesso em: 10 out. 2017.

28. WHO. World Health Organization. 2014. Suicide prevention. Department of Mental Health. Geneva. Disponivel em: http://www.who.int/mental health/prevention/suicide/suicideprevent/es/index.html. Acesso em: 17 dez. 2017.

29. WOODHEAD C, et al. Mental health among UK inner city non-heterosexuals: The role of risk factors, protective factors and place. Rev. Epidemiology and Psychiatric Sciences. 2016;25(5):450-46. 